Supporting Information

\title{
From Slow to Fast Self-Healing at Ambient Temperature of High-Modulus Reversible Poly(methacrylate) Networks. Single- and Dual-Dynamics and the Effect of Phase-Separation.
}

\author{
D. Ehrhardt ${ }^{a}$, J. Mangialetto ${ }^{a^{*}}$, K. Van Durme ${ }^{a, b}$, B. Van Mele ${ }^{a}, N$. Van den Brande ${ }^{a}$
}

aPhysical Chemistry and Polymer Science (FYSC), Vrije Universiteit Brussel (VUB), Pleinlaan 2, 1050 Brussels, Belgium

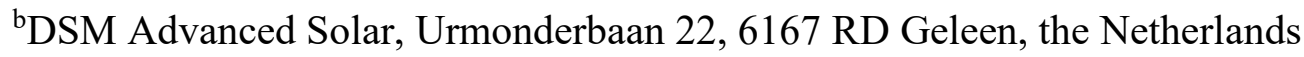

*E-mail: Jessica.Mangialetto@vub.be

Synthesis of Methacrylate-Functionalized Furans and Bismaleimide

\section{Methacrylate-functionalized Furan F1}

1 equivalent FI and 1 equivalent PPG375-MA are mixed, with about $1 \mathrm{wt} \%$ DBTDL as catalyst. The urethane reaction is complete after $15 \mathrm{~min}$.

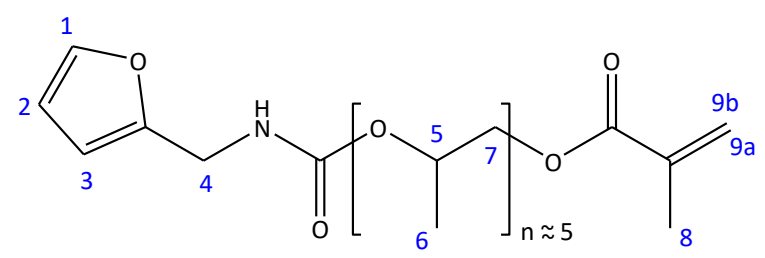

F1: ${ }^{1} \mathrm{H}$ NMR [ppm], 7.42-7.28 (1H, s, H1), 6.36-6.16 (2H, m, H2 and H3), 6.16-6.02 (1H, m, H9b), 5.63-5.46 (1H, m, H9a), 4.44-4.22 (2H, m, H4), 4.22-3.17 (16H, m, H5 and H7), 2.00-1.84 $(3 \mathrm{H}, \mathrm{m}, \mathrm{H} 8), 1.3-1.00(17 \mathrm{H}, \mathrm{m}, \mathrm{H} 6)$.

\section{Methacrylate-functionalized Furan F2}

FA and IEMA are mixed in equimolar amounts, with about $1 \mathrm{wt} \%$ DBTDL as catalyst. The reaction is complete after $5 \mathrm{~min}$. 


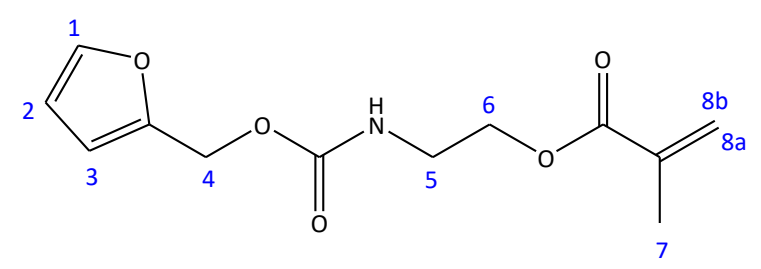

F2: ${ }^{1} \mathrm{H}$ NMR [ppm], 7.47-7.39 (1H, s, H1), 6.47-6.32 (2H, m, H2 and H3), 6.16-6.06 (1H, m, H8b), 5.64-5.54 (1H, m, H8a), 5.14-4.98 (2H, s, H4), 4.30-4.14 (2H, m, H6), 3.61-3.42 (2H, m, H5), 1.99-1.89 (3H, m, H7).

\section{Methacrylate-functionalized Furan F3}

FA and IPDI are mixed in equimolar amounts, using about $1 \mathrm{wt} \%$ DBTDL as catalyst, and allowed to react overnight. Then, 1 equivalent HEMA is added. The urethane reaction is complete after $7 \mathrm{~h}$.

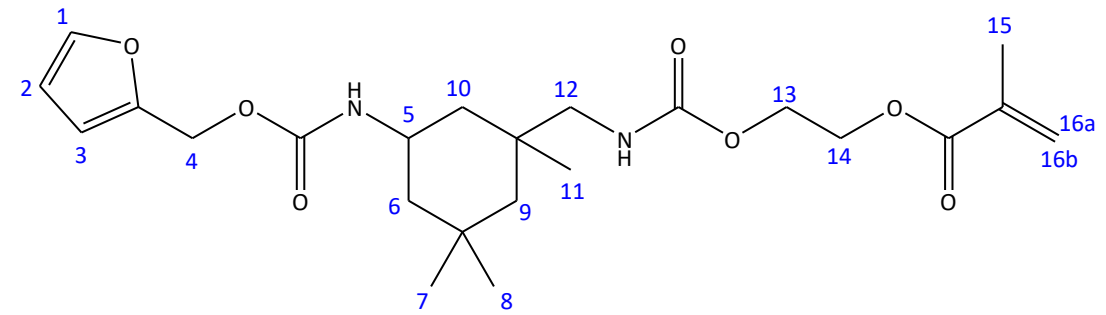

F3: ${ }^{1} \mathrm{H}$ NMR [ppm], 7.49-7.37 (1H, s, H1), 6.48-6.30 (2H, m, H2 and H3), 6.19-6.10 (1H, m, H16b), 5.67-5.55 (1H, m, H16a), 5.12-4.97 (2H, m, H4), 4.39-4.29 (4H, m, H13 and H14), 3.953.28 (1H, m, H5), 3.01-2.83 (2H, m, H12), 2.06-1.57 (7H, m, H6, H10 and H15), 1.13-0.90 (11H, $\mathrm{m}, \mathrm{H} 7, \mathrm{H} 8, \mathrm{H} 9$ and $\mathrm{H} 11)$.

\section{Bismaleimide M1}

1 equivalent dried (over $4 \AA$ molecular sieves), bishydroxyl-functionalized polyether (PPO-1000) and 1 equivalent IPDI are allowed to react for $6 \mathrm{~h}$, under the addition of about $1 \mathrm{wt} \%$ DBTDL as catalyst. Subsequently, 1 equivalent melted 2-HEM is added. The urethane reaction is complete after 10 days.

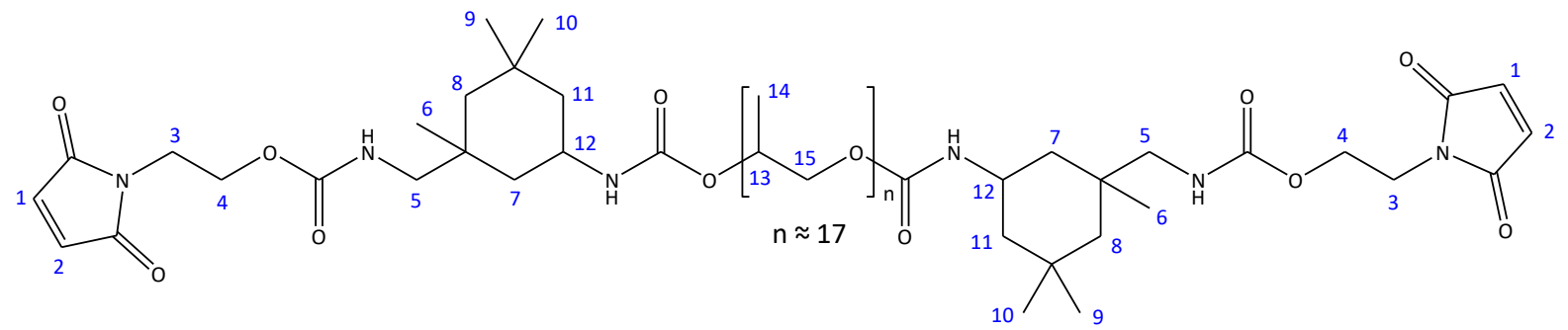

M1: ${ }^{1} \mathrm{H}$ NMR [ppm], 6.82-6.68 (4H, s, H1 and H2), 4.33-2.76 (74H, m, H3, H4, H5, H12, H13 and $\mathrm{H} 15), 1.98-1.33$ (8H, m, H7 and H11), 1.33-0.90 (85H, m, H6, H8, H9, H10 and H14). 
Table S1. Properties of methacrylate-functionalized furans F1, F2, F3 and maleimide M1. All $\mathrm{T}_{\mathrm{g}}$ 's are determined by DSC.

\begin{tabular}{ccccc} 
No. & $\mathbf{f}_{\text {furan }} / \mathbf{m a l e i m i d e}$ & $\mathbf{M}\left[\mathbf{g ~ m o l}^{-1} \mathbf{]}\right.$ & $\mathbf{T}_{\mathbf{g}}\left[{ }^{\circ} \mathbf{C}\right]$ & $\mathbf{T}_{\mathbf{m}}\left[{ }^{\circ} \mathbf{C}\right]$ \\
\hline F1 & 1 & 498 & -57 & - \\
\hline F2 & 1 & 253 & -53 & - \\
\hline F3 & 1 & 450 & -17 & - \\
\hline M1 & 2 & 1726 & -22 & - \\
\hline
\end{tabular}

$\underline{\text { Synthesis of Irreversible Methacrylates }}$

\section{Tris-methacrylate prepolymer I1}

PPG375-MA is mixed with 1 equivalent IPDI, using about $1 \mathrm{wt} \%$ DBTDL as catalyst, and allowed to react for $6 \mathrm{~h}$. Subsequently, an equimolar amount of Daltolac R630 is added. The reaction is complete after 7 days.

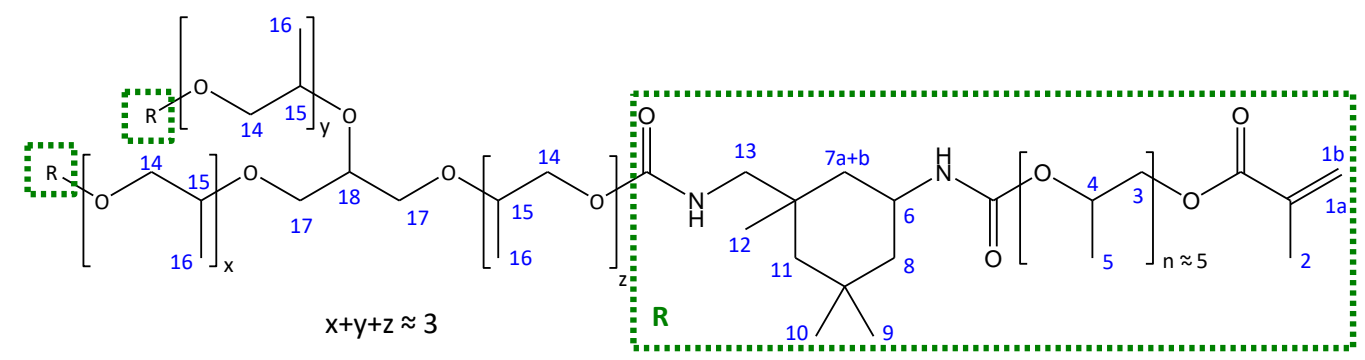

I1: ${ }^{1} \mathrm{H}$ NMR [ppm], 6.21-6.05 (3H, m, H1b), 5.64-5.51 (3H, m, H1a), 4.33-2.73 (65H, m, H3, H4, H6, H13, H14, H15, H17 and H18), 2.04-1.90 (9H, m, H2), 1.90-1.56 (8H, m, H7 and H8), 1.30-1.02 (74H, m, H5, H9, H10, H11, H12 and H16).

\section{Bis-methacrylate prepolymer I2}

PPO-2700 is allowed to react for $6 \mathrm{~h}$ with 1 equivalent IPDI and $1 \mathrm{wt} \%$ DBTDL as catalyst. Then, an equimolar amount of HEMA is added. The reaction is complete after 2 weeks.

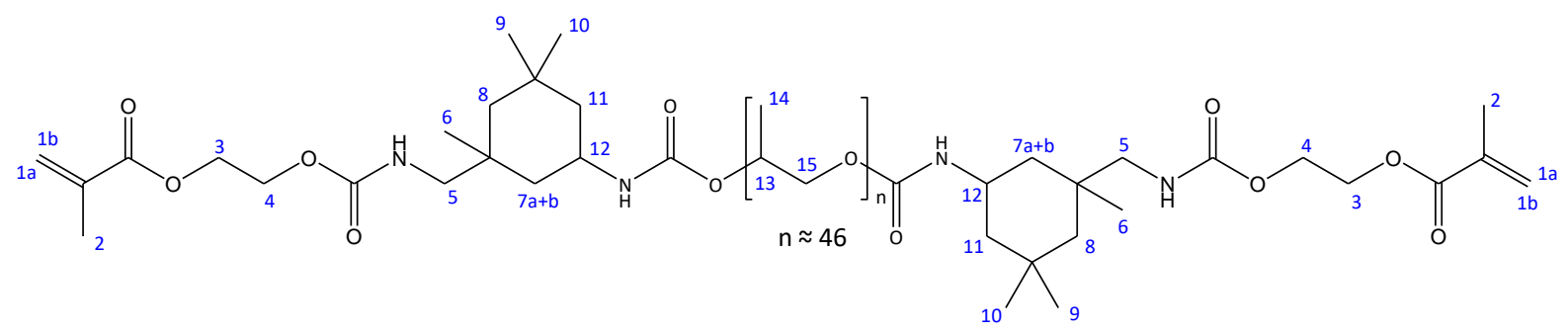

I2: ${ }^{1} \mathrm{H}$ NMR [ppm], 6.23-6.09 (2H, m, H1b), 5.68-5.54 (2H, m, H1a), 4.44-2.79 (174H, m, H3, H4, H5, H12, H13 and H15), 2.70-2.07 (14H, m, H2, H7 and H11), 1.31-0.91 (181H, m, H6, H8, $\mathrm{H} 9, \mathrm{H} 10$ and H14). 


\section{Phase-Separated Poly(methacrylate) Networks}

(a)

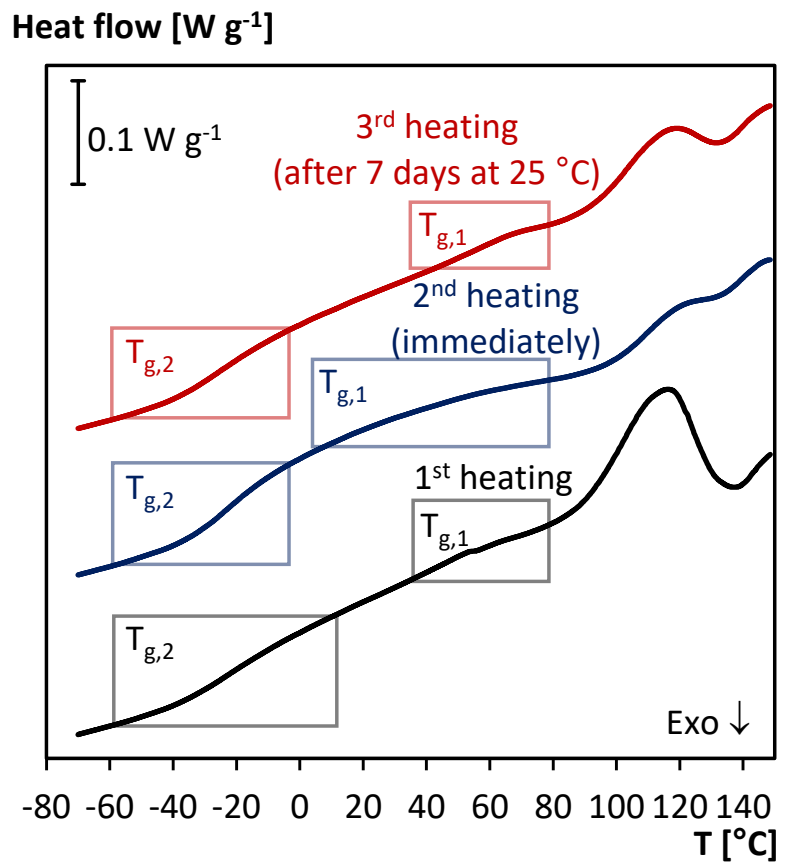

(b)

\section{Heat flow $\left[\mathrm{W} \mathrm{g}^{-1}\right]$}

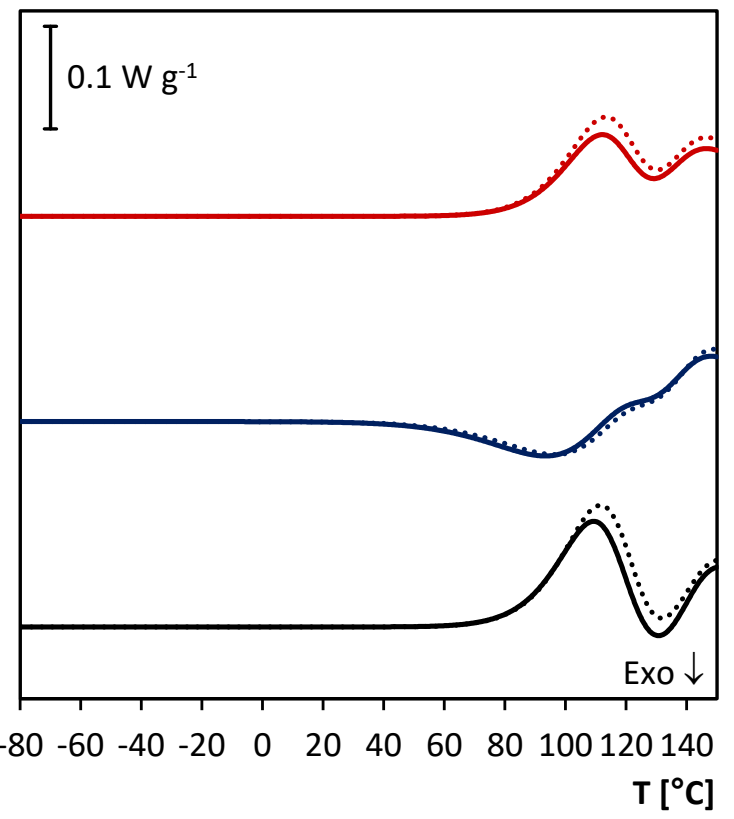

Figure S1. (a) DSC thermogram of RHM4. Black line: $1^{\text {st }}$ heating, blue line: $2^{\text {nd }}$ heating (immediately after $1^{\text {st }}$ heating), red line: $3^{\text {rd }}$ heating (after 7 days at $25^{\circ} \mathrm{C}$ ). Frames indicate $\mathrm{T}_{\mathrm{g}}$ region. (b) Simulated normalized heat flows of $1^{\text {st }}, 2^{\text {nd }}$ and $3^{\text {rd }}$ heating (without $T_{g}$ ), showing the effect of ongoing Diels-Alder reactions, using the kinetic parameters of ref ${ }^{19}$. Full lines: phase separation taken into account, dotted lines: simulations for homogeneous system (see text). Curves vertically shifted for clarity. 
(a)

\section{Heat flow [ $\left.\mathrm{W} \mathrm{g}^{-1}\right]$}

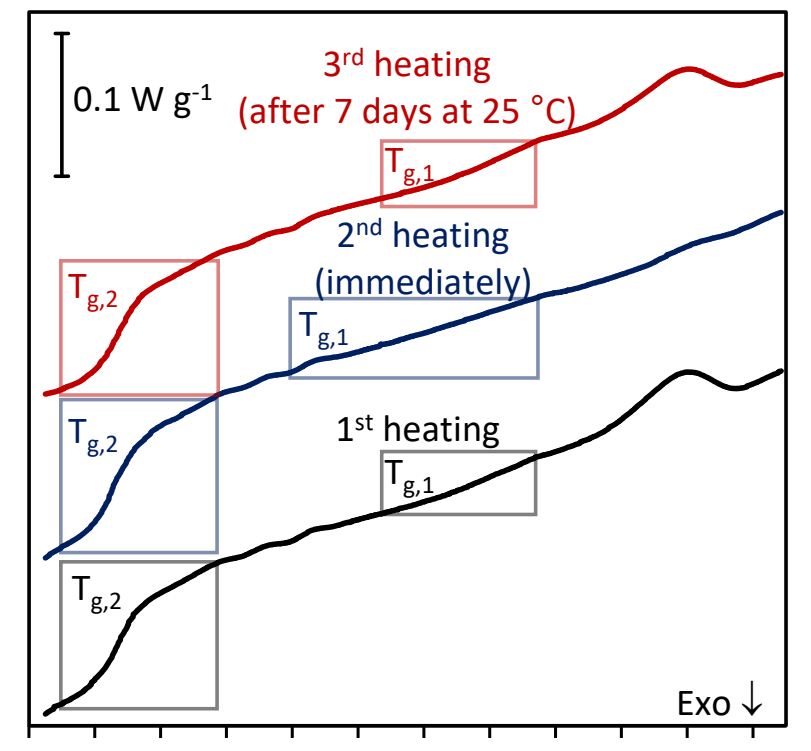

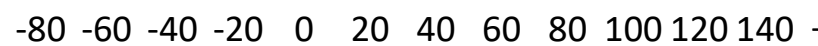

$\mathrm{T}\left[{ }^{\circ} \mathrm{C}\right]$ (b)

\section{Heat flow $\left[\mathrm{W} \mathrm{g}^{-1}\right]$}

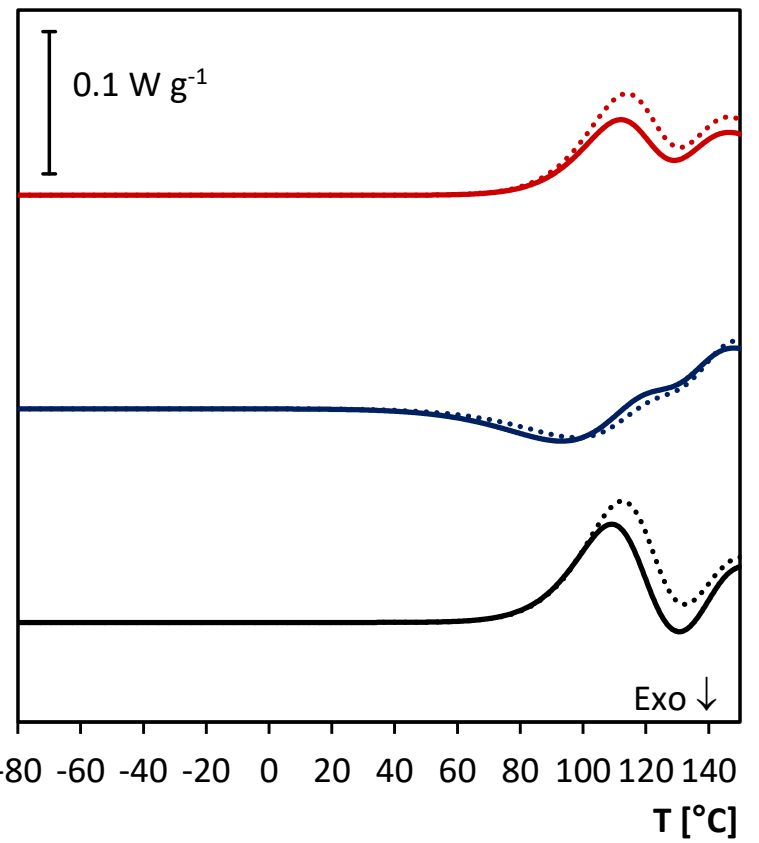

Figure S2. (a) DSC thermogram of RLM2. Black line: $1^{\text {st }}$ heating, blue line: $2^{\text {nd }}$ heating (immediately after $1^{\text {st }}$ heating), red line: $3^{\text {rd }}$ heating (after 7 days at $25^{\circ} \mathrm{C}$ ). Frames indicate $\mathrm{T}_{\mathrm{g}}$ region. (b) Simulated normalized heat flows of $1^{\text {st }}, 2^{\text {nd }}$ and $3^{\text {rd }}$ heating (without $T_{\mathrm{g}}$ ), showing the effect of ongoing Diels-Alder reactions, using the kinetic parameters of ref ${ }^{19}$. Full lines: phase separation taken into account, dotted lines: simulations for homogeneous system (see text). Curves vertically shifted for clarity.

$\underline{\text { H-bonding to Support Diels-Alder Reversible Bonding }}$

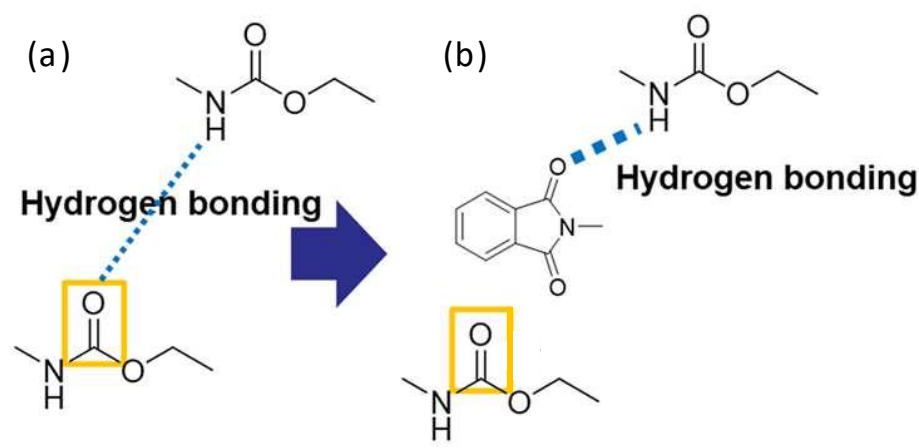

Figure S3. Schematic illustration of hydrogen bonding between (a) two urethane groups and (b) a urethane group and an imide group. Adapted from ${ }^{23}$. 
$\underline{\text { Phase-Separated High-Modulus Reversible Poly(methacrylate) Networks }}$

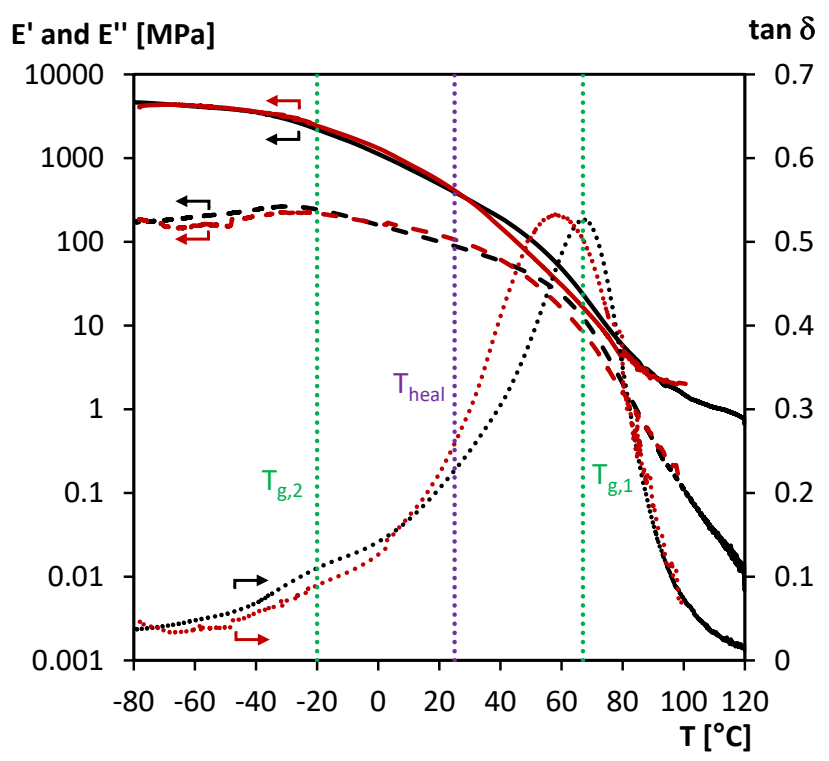

Figure S4. DMA of RHM4, measured on a film in tension mode (undamaged, black) and on a powder rectangular bar in 3-point bending mode (healed for $0 \mathrm{~h}$ at $\mathrm{T}_{\text {heal }}=25^{\circ} \mathrm{C}$ after damage, red). Storage modulus E' (full lines), loss modulus E', (dashed lines) and tan $\delta$ (dotted lines). 
Interplay between Hydrogen Bonding and Local Mobility in Poly(methacrylate) Networks with $\underline{\text { Reversible Diels-Alder Crosslinks }}$

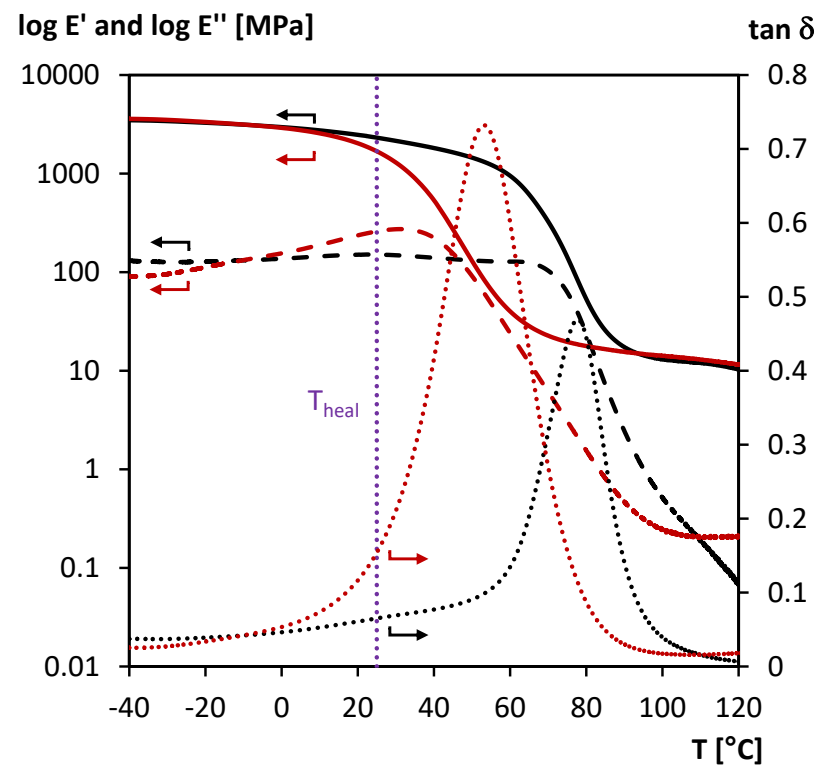

Figure S5. DMA of RHM1 network (black) and of RHM2 network (red), measured on a film in tension mode. Storage modulus E' (full lines), loss modulus E', (dashed lines), $\tan \delta$ (dotted lines).

Table S2. Initial storage modulus E' at $25^{\circ} \mathrm{C}$, relative damage and damage recovery after $10 \mathrm{~min}$ of RLM1, RLM2 and ILM1.

\begin{tabular}{|c|c|c|c|c|c|}
\hline \multicolumn{2}{|c|}{ Sample } & & $\begin{array}{c}\mathbf{E} \\
{[\mathbf{M P a}]}\end{array}$ & $\begin{array}{c}\text { relative damage } \\
{[\%]}\end{array}$ & $\begin{array}{c}\text { damage recovery } \\
{[\%]}\end{array}$ \\
\hline \multirow[t]{3}{*}{ RLM1 } & \multirow[t]{3}{*}{ Sample 1} & Initial & 6.89 & 0 & - \\
\hline & & Damaged & 4.76 & 31 & - \\
\hline & & 10 min healing & 4.85 & 30 & 4 \\
\hline \multirow[t]{3}{*}{ RLM1 } & \multirow[t]{3}{*}{ Sample 2} & Initial & 6.92 & 0 & - \\
\hline & & Damaged & 5.55 & 20 & - \\
\hline & & 10 min healing & 5.57 & 19 & 1 \\
\hline \multirow[t]{3}{*}{ RLM1 } & \multirow[t]{3}{*}{ Sample 3} & Initial & 6.91 & 0 & - \\
\hline & & Damaged & 2.24 & 68 & - \\
\hline & & 10 min healing & 2.23 & 68 & 0 \\
\hline
\end{tabular}




\begin{tabular}{|c|c|c|c|c|c|}
\hline \multirow[t]{3}{*}{ RLM1 } & \multirow[t]{3}{*}{ Sample 4} & Initial & 6.88 & 0 & - \\
\hline & & Damaged & 4.53 & 34 & - \\
\hline & & 10 min healing & 4.65 & 32 & 5 \\
\hline \multirow[t]{3}{*}{ RLM2 } & \multirow[t]{3}{*}{ Sample 1} & Initial & 12.06 & 0 & - \\
\hline & & Damaged & 10.87 & 10 & - \\
\hline & & 10 min healing & 11.44 & 5 & 48 \\
\hline \multirow[t]{3}{*}{ RLM2 } & \multirow[t]{3}{*}{ Sample 2} & Initial & 10.53 & 0 & - \\
\hline & & Damaged & 7.50 & 29 & - \\
\hline & & 10 min healing & 8.27 & 21 & 25 \\
\hline \multirow[t]{3}{*}{ RLM2 } & \multirow[t]{3}{*}{ Sample 3} & Initial & 9.46 & 0 & - \\
\hline & & Damaged & 6.40 & 32 & - \\
\hline & & 10 min healing & 7.50 & 21 & 36 \\
\hline \multirow[t]{3}{*}{ RLM2 } & \multirow[t]{3}{*}{ Sample 4} & Initial & 10.76 & 0 & - \\
\hline & & Damaged & 9.43 & 12 & - \\
\hline & & 10 min healing & 9.87 & 8 & 33 \\
\hline \multirow[t]{3}{*}{ ILM1 } & \multirow[t]{3}{*}{ Sample 1} & Initial & 6.90 & 0 & - \\
\hline & & Damaged & 5.53 & 20 & - \\
\hline & & 10 min healing & 5.77 & 16 & 18 \\
\hline \multirow[t]{3}{*}{ ILM1 } & \multirow[t]{3}{*}{ Sample 2} & Initial & 6.67 & 0 & - \\
\hline & & Damaged & 5.51 & 17 & - \\
\hline & & 10 min healing & 5.62 & 16 & 9 \\
\hline \multirow[t]{3}{*}{ ILM1 } & \multirow[t]{3}{*}{ Sample 3} & Initial & 7.12 & 0 & - \\
\hline & & Damaged & 6.13 & 14 & - \\
\hline & & 10 min healing & 6.07 & 15 & 6 \\
\hline
\end{tabular}

